\title{
HEART RATE VARIABILITY CLASSIFICATION USING SADE-ELM CLASSIFIER WITH BAT FEATURE SELECTION
}

\author{
R. Kavitha ${ }^{1}$ and T. Christopher ${ }^{2}$ \\ ${ }^{1}$ Department of Computer Science, PSGR Krishnammal College for Women, India \\ ${ }^{2}$ Department of Computer Science, Government Arts College, Coimbatore, India
}

\begin{abstract}
The electrical activity of the human heart is measured by the vital bio medical signal called ECG. This electrocardiogram is employed as a crucial source to gather the diagnostic information of a patient's cardiopathy. The monitoring function of cardiac disease is diagnosed by documenting and handling the electrocardiogram (ECG) impulses. In the recent years many research has been done and developing an enhanced method to identify the risk in the patient's body condition by processing and analysing the ECG signal. This analysis of the signal helps to find the cardiac abnormalities, arrhythmias, and many other heart problems. ECG signal is processed to detect the variability in heart rhythm; heart rate variability is calculated based on the time interval between heart beats. Heart Rate Variability HRV is measured by the variation in the beat to beat interval. The Heart rate Variability $(H R V)$ is an essential aspect to diagnose the properties of the heart. Recent development enhances the potential with the aid of non-linear metrics in reference point with feature selection. In this paper, the fundamental elements are taken from the ECG signal for feature selection process where Bat algorithm is employed for feature selection to predict the best feature and presented to the classifier for accurate classification. The popular machine learning algorithm ELM is taken for classification, integrated with evolutionary algorithm named SelfAdaptive Differential Evolution Extreme Learning Machine SADEELM to improve the reliability of classification. It combines Effective Fuzzy Kohonen clustering network (EFKCN) to be able to increase the accuracy of the effect for HRV transmission classification. Hence, it is observed that the experiment carried out unveils that the precision is improved by the SADE-ELM method and concurrently optimizes the computation time.
\end{abstract}

\section{Keywords:}

Self-adaptive Differential Evolution, Extreme Learning Machine, Bat Algorithm, Efficient Fuzzy Kohonen Clustering Network

\section{INTRODUCTION}

Heart problem is a serious issue, where many people affect from this risk factor due to unhealthy food, obesity, diabetes, hypertension, blood pressure and physical inactivates. Each year consistently, 9.7 million passings are ascribed to coronary heart diseases, which includes $52 \%$ of dying is because of strokes and $47 \%$ of deaths are because of heart illnesses. Heart disease is usually diagnosed after a risk assessment and further test with the patient. In which the ECG is an important and common test to find the normal and abnormal activities of the heart and to detect the problem in the heart. The electrical power activity associated with any muscle action moves through various cells and eventually extends to the top of the body where it could be recognized and diagnosed by electrodes put on the skin of the patient's chest, arms, and legs. ECG (Electrocardiogram) gives enormous information of the structure and functioning of the heart. This ECG is mostly used to detect the various abnormalities in the
Heart Rhythm, the size of the heart chamber and the damage to the heart muscles. Hence, this ECG is a test which records the electrical activity of the heart and assesses the Heart rate variability i.e., the rhythm of the heart. The mind directs the physical body by controlling and taking care of the heart and various organs through the autonomic anxious system. This physiological variety of heart rate, controlled via autonomic sensory system is named HRV (Heart Rate Variability). ECG can be used as a simple for calculating the heart rate variability. Heart rate variability (HRV) is computed by the amount of time in milliseconds between two heartbeats. HRV fluctuates as you take breathe in and out. This HRV is generally new strategy and popular parameter in the field of cardiology. Many types of research are done using this HRV to find the normal and abnormal activity of the heart rhythm. Calculating the heart rate variance unveils the extensive selection of information about the human individuals and body. The heart is influenced by the autonomic nervous system which can increase or decrease the heart rate in line with the requirements of the body. The ECG signal consists of sequence waves associated with each beat as P-QRS and T wave. This detection of ECG wave is an important factor to diagnosis cardiac disease. The accurate performance of these important factors is applied to the HRV signal for denoising and the classification rule is employed in the testing dataset.

HRV evaluation can be viewed as an important diagnostic tool in cardiology. A few examples of computerized arrhythmia detection and classification methods which were analyzed ECG transmission using neural networks wavelet transforms is utilized [1]. The classifier support vector machines were examined for ECG signal classification [2]. Fuzzy logic is employed for signal classification [3] and the rule-based algorithms is employed in arrhythmia classification system based on the RR-interval signal knowledge-based way for arrhythmia classification into four different categories was proposed by [4]. Alternatively the RR time intervals are less afflicted by the noise, analysis of HRV transmission is a far more powerful method. Different HRV sign evaluation methods for arrhythmia classification were unveiled [5]. In [6] classify the insight HRV sections into different arrhythmia classes using Fuzzy Kohonen Clustering Network to predict the interval between the data used. In [7] morphological features are extracted for specialized medical observation of heart diseases. The feature removal using traditional techniques generally deliver a big range of features, and several might be insignificant. Therefore, the normal practice is to draw out key features useful in the classification. This paper reveals a feature removal method rather than using traditional [8] feature extraction techniques.

In this paper, feature removal is dependent on extracting key features using aspect influenced algorithm called Bat algorithm 
[9]. Prior studies extracted the main element features using statistical and morphological techniques like Combination Wavelet transform (WT) [10], Morphological features and SVM, Multiple Learning (MIL) [11] which give less accuracy and reliability in the classification of HRV. To discover the best local minimum illustrations for the type inspired search engine optimization algorithms are ant colony [12], particle swarm [13], cuckoo search, bacterial foraging optimization (BFO) [14]. Bat Algorithm is a particular category of swarm intellect used for extracting features. A couple of two considerations to be well balanced in the type inspired meta-heuristic algorithms they really simply exploitation and exploration. The earlier studies [15] possessed the good thing about exploration approach first and exploitation strategy later. The Fig.1 shows the information regarding ECG signal. The $\mathrm{P}$ wave is the first wave moved in the upward direction of the ECG detection. The R wave is a larger upward deflection. The RR interval is the measurement of the time between the R-wave of one heartbeat and the $\mathrm{R}$ wave of the preceding heartbeat. The $\mathrm{R}$ wave is the first upward deflection after the $\mathrm{P}$ wave. The $\mathrm{R}$ wave is normally the easiest waveform to identify on the ECG and represents early ventricular depolarization. HRV is calculated by the mean value of RR interval and the deviation is measured.

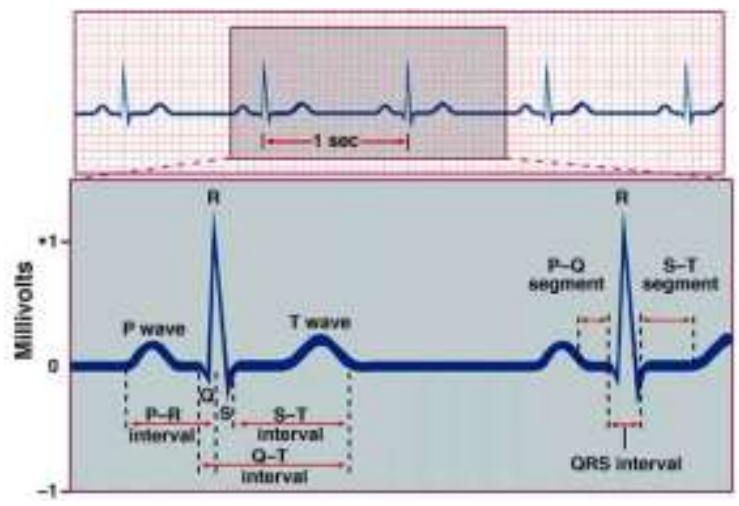

Fig.1. ECG signal with PQRST wave and segment

In this work, the HRV is given as input data to classify the heart rate three main steps are utilized such as pre-processing, feature extraction and feature selection is performed using bat algorithm which gives more accurate key feature selection for clustering the data and the clustered data is produced to the SADE-ELM classifier which gives the result. In this paper, an efficient HRV-based arrhythmia classification method is presented in order to reduce the learning time and also to increase the efficiency of the classifier. In the proposed work, SelfAdaptive Differential Evolution Extreme Learning Machine (SADE-ELM) uses bat behaviour to choose features to improve the reliability of classification. It combines Effective Fuzzy Kohonen Clustering Network (EFKCN) to be able to increase the accuracy of the effect for HRV transmission classification.

The paper goes in the following structure; section 2 describes the materials and methods. Section 3 explains the Bat algorithm frame work. In section 4 the clustering of EFKCN is discussed. Section 5 gives the description of Classifier. Section 6 has experimental result and discussion proceeding by the conclusion in section7.

\section{METHODS AND MATERIALS}

The required data are collected from the MIT-BIH arrhythmia database. The available data set is used for the evaluation of the arrhythmia detectors. Heart rate variability is the physiological occurrence of deviation in enough time periods between heartbeats. The best method to find the beat is ECG which gives clear waveform. The proposed method has to pre-process the signal as the first step. HRV or Heart rate variability is the measurement of the time interval between each successive heart beat to beat, which is known as R-R interval or (N-N internal). The heartbeat is not a fixed or constant for all people the beat varies for each and every moment hence the term variability is used. By measuring the heart beat gives a lot of information of the patient's body condition and heart. Many methods are adopted to measure the heart beat such as ECG, Blood pressure, PPG (photoplethysmography) and ballistocardiogram. Among these ECG is used widely which gives a clear waveform to measure the heart rate of the patient to find the abnormalities. The decreased HRV and abnormal HRV signify the patient risk of Cardiac arrhythmia or irregular heartbeat. Hence this HRV analysis is used as a tool and applied to find the risk of the patients suffered from the cardiac disorder. For the analysis and assessment of HRV, the commonly used method is linear and nonlinear methods. The proposed architecture diagram is shown in Fig.2.

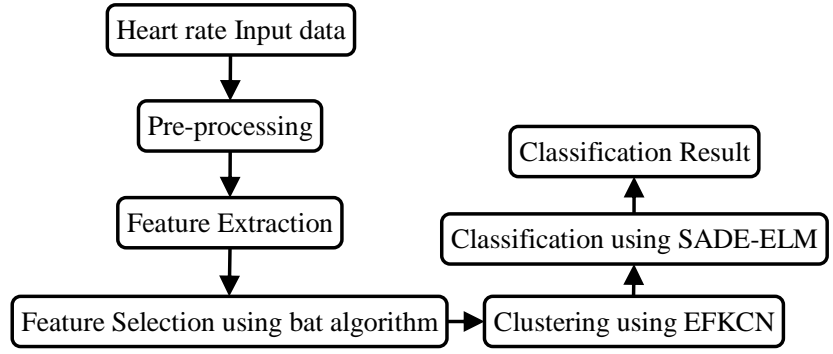

Fig.2. Proposed Architecture for Classification

\subsection{LINEAR AND NONLINEAR METHODS}

Time domain and Frequency domain methods is a linear analysis and the widely used non-linear method analysis is Poincare Plot graph. There are three different rhythms such as HF (High Frequency) ranges $0.25 \mathrm{~Hz}$ which is measured 15 cycles per minute and LF (Low Frequency ranges $0.1 \mathrm{~Hz} 6$ cycles per minute) and VLF(very Low Frequency ranges $0.016 \mathrm{~Hz} 1$ cycle per minute).

\subsubsection{Time Domain:}

The Time-domain method has the following variables, Mean $H R$ : The mean value of the heart rate within about a minute in each section. Instantaneous heart rate beat each and every minute is add up to 60 divided by each R-R period (second). NN50: The $\mathrm{NN}$ is the number of pairs of successive beat intervals that differ by more than $50 \mathrm{~ms}$. STD HR: The typical deviation of Instantaneous heart rate in each section. pNN50: The quantity of successive difference of $64 \mathrm{R}-\mathrm{R}$ intervals that differs more than $50 \mathrm{~ms}$, respectively, divided by 64 and $\mathrm{P}$ is the proportion.

\subsubsection{Frequency Domain:}

In Frequency domain analysis many methods are adopted and the widely used is PSD (Power Spectral Density) utilizes the parametric and non-parametric method which gives the data on power distribution based on the frequencies. This method has the 
following variables, Total Power: It predicts the sum of all frequency domain components. Low-Frequency power $(L F)$ : It has 3-9 cycles per minute bar receptor influences on HR. HighFrequency power (HF): HF has 9-24 cycles per minute, respiratory sinus arrhythmia.

\subsubsection{Nonlinear Analysis:}

Many methods are adopted under nonlinear analysis to get a relevant and accurate data information the widely used nonlinear method of HRV analysis is Poincare plot. SD1/SD2: Poincare plot is a graphical representation of the correlation between successive $R R$ intervals. This is obtained plotting each $\mathrm{RR}$ interval, $R R(n+1)$ as a function of the previous interval, $R R(n)$ in $R R$ interval time series. This plot is quantitatively analyzed by calculating the standard deviation of the distances of the time series points from the lines $y=x$ and $y=x+2 R R_{m}$, in which $2 R R_{m}$ is the mean of all values of RR interval time series. These values are named $S D 1$ and $S D 2$, respectively. In fact, $S D 1$ represents the fast beat-to-beat variability, while $S D 2$ describes the relatively long-term variability in the HRV signal. In this work, SD1/SD2 is used as the first nonlinear feature which is extracted from HRV segments.

\subsubsection{SpEn:}

The Spectral Entropy shows the complexness of the insight time series (HRV section) in the rate of recurrence domain. Large ideals of $S p E n$ show high irregularity and smaller ideals of it reveal more regular time series. The Shannon's route entropy is employed to calculate the spectral entropy shown in Eq.(1) of the procedure. Heuristically, the entropy is interpreted as a way of measuring uncertainty about the function of $f$. Thus entropy can be utilized as a way of measuring system complexness. The spectral entropy describes the complexity of the HRV.

$$
S p E n=-\sum_{f} p_{f} \log p_{f}
$$

where, $p_{f}$ is the PDF (possibility density function) value at a rate of occurrence $f$.

Noise immunity is the major asset in all electronic device. Before processing the actual signal the removal of unwanted noise is one of the important methods. The HRV data used in this work is generated from the ECG signals where the input ECG data is taken from the MIT-BIH arrhythmia database.

\subsection{PRE-PROCESSING}

The HRV signal is extracted from the ECG signals. Naturally many interfering signals such as Power line interference, Electrode contact noise, baseline drift and Electrode contact noise, baseline drift and motion artifacts can affect the extraction process. Hence, these signal noise can be removed by using 5$15 \mathrm{~Hz}$ band pass filter. The threshold value is used to process the signal and the resultant signal is identified as HRV signal.

\subsection{FEATURE EXTRACTION}

The analysis of a large number of variables requires a large amount of memory and computational power. When carrying out analysis of sophisticated data one of the major problems is due to the amount of variables involved. This feature extraction is used to extract the relevant feature in order to find the abnormality in the heart disorder. Hence we need a strong model to extract the parameters.

\subsection{FEATURE SELECTION}

Feature selection is proposed to select the subset of the most relevant feature to minimize the error rate. Here the feature selection is carried out with bat algorithm which gives the most relevant data as input for clustering and classifying the data to produce the accurate result.

\section{FEATURE SELECTION USING BAT ALGORITHM}

The bat-inspired algorithm is a metaheuristic enhancement algorithm. The bat algorithm is applied in almost every area of optimization, classification and feature selection. This bat algorithm is based on the echolocation behaviour of micro bats with capricious beating ante of discharge and loudness. The idealization of the echolocation of micro bats can be summarized as:

- Each exclusive bat flies arbitrarily with a speed $v_{i}$ at position (solution) $x_{i}$ with a differing rate of occurrence or wavelength and loudness $A_{i}$.

- Since it searches and confirms its victim, it changes occurrence, pulse, and loudness, emission rate r. Search is intensified by an area random walk.

- A collection of the best continues until certain stop conditions are fulfilled.

This essentially runs on the frequency tuning strategy to control the powerful behaviour of the swarm of bats, and the balance between exploration and exploitation can be manipulated by tuning algorithm-dependent parameters in bat algorithm.

The customary feature extraction methods yield a huge number of features generally, and several might be insignificant. Therefore, the effective approach in this research analysis is to draw out the main element features useful in the classification of ECG beats. With this sub-section 30 key features from 300 samples are extracted using bat algorithm. Bats [16] will be the fascinating band of birds. They count on echolocation which has a total of 1200 varieties of bats [17]. Over fifty percent of these rely on echolocation to find their prey. Bats echolocation capacity usually, a number of the bats use a superior and sophisticated sense ability to hear. The noises are released that bounces echo back again from the pests or the things in their route. From these echoes, bats can identify the pests or thing lengths which are using their current position and also estimate how big are insects or things within a portion of the second [18].

\section{FRAMEWORK OF BAT ALGORITHM}

(a) Initialization: The fitness of the original inhabitants is assessed using the Rosenbrock work as shown in the Eq.(2) and the ideals of pulse rate $r_{i}$, loudness $A_{i}$ and rate of consistency $f_{\min }, f_{\max }$ are initialized as shown in Eq.(3), where, $\mathrm{j}$ is the dimensions and $x(i)$ is the $i^{\text {th }}$ bat.

$$
\begin{gathered}
f(x)=\sum_{i=1}^{j-1}\left[100\left(x_{i}+1-x_{i}^{2}\right) x^{2}+\left(x_{i}-1\right)^{2}\right] \\
f_{i}=f_{\min }+\delta\left(f_{\max }-f_{\min }\right)
\end{gathered}
$$


(b) The activity of Virtual Bats: The brand new inhabitants people is made by modifying the position $x_{i}$ and the speed $v_{i}$ for each and every bat in the populace as given in Eq.(5) and Eq.(6) The speed and position improvements of the $i^{\text {th }}$ bat are determined and computed using Eq.(5) and Eq.(6). The wavelength and loudness are assorted in line with the location and size of the food. The $v_{i}$ and $x_{i}$ are initialized with some preliminary random ideals and a fitness function $f$ is determined, using the contaminants positional coordinates as source values. The very best fitness value among all the bats is denoted as $\mathrm{x}_{\text {gbest. }}$. The difference between $x_{i}^{(t)}$ and $x_{\text {gbest }}$ is an optimistic value this means $x_{\text {gbest }}$ bat has more quantity of features than those of $i^{\text {th }}$ bat. This difference is summed up with the prior speed to accelerate the activity of $i^{\text {th }}$ bat towards $x_{\text {gbest }}$ bat. When the difference is negative, this means $i^{\text {th }}$ bat has more quantity of features than those of $x_{\text {gbest }}$ bat. This difference is added to the prior velocity to diminish the speed of the $i^{\text {th }}$ bat.

$$
\begin{gathered}
f_{i}=\frac{v_{i}}{\lambda_{i}} \\
v_{i}^{t}=v_{i}^{t-1}+\left(X_{i}^{t}-X_{\text {gbest }}^{t}\right) f_{i} \\
X_{i}^{t}=X_{i}^{t-1}+v_{i}^{t}
\end{gathered}
$$

Bat algorithm local search capacity: To be able to improve the local search capacity for the algorithm, [18] has generated the best answer using the Eq.(7) where $x_{\text {gbest }}$ is a superior quality solution chosen by some system. $A^{t}$ is the common loudness value of all bats at $t^{\text {th }}$ time step and is made by some arbitrary mechanism varying between 1 and 1 .

$$
X_{\text {new }}=X_{\text {old }}+\rho A^{t}
$$

(c) Loudness $(A)$ : Loudness $A_{i}$ has been modified using Eq. (8) as the iterations continue. As bats deal with their food, the loudness usually diminishes. The quantity of decrease depends upon $\alpha$ where $0<\alpha<1$. Generally, the loudness value will reduce when the bat begins nearing the best solution as shows in the Eq.(8) and Eq.(9).

$$
\begin{gathered}
A_{i}^{t+1}=\alpha A_{i}^{t} \\
r_{i}^{t+1}=r_{i}^{0}\left[1=e^{-\gamma t} A_{i}(t+1)\right]=\alpha A_{i}(t)
\end{gathered}
$$

Emission of pulse rate (r): By using Eq.(9) the pulse emission rate $r_{i}$ has been modified as the bats deal with their food $r$ rises and pulse rate $r$ yields an improved solution near $X_{\text {gbest }}$, higher pulse rate does not produce the best answer in local search space. Using Eq.(9) where $\gamma$ is greater than 0 and $A$ are updated then only the new resolution is created higher than the previous solution, where $\gamma$ is constant. The bat algorithm is much superior to other algorithms in conditions of accuracy and efficiency. The use of bat algorithm is mainly for the following purposes:

- Calculating the ideal and most effective value of the given fitness function.

- Reducing feature group of a population.

The algorithm is being employed by us for the feature reduction so that the best and relevant feature can be found. Here
30,873 beats are taken and loaded into MATLAB command as " $X=$ xlsread" to read the values.

$X$ row signifies the features and the columns of $X$ signifies how big population is. Use Eq.(1) to calculate the fitness function for the given population. The best value is predicted and stored by giving the minimum value of the fitness among all the populace. Now, move the populace to a fresh location to determine and identify the local solution throughout to predict the best solution by using the Eq.(10).

$$
f_{t}=f_{\text {min }}+\left(f_{\text {max }}-f_{\text {min }}\right) \times \text { rand }
$$

In this proposed work, the best features are selected using the bat algorithm and this feature is given as input to the classifier. The following steps are used to find the relevant feature. The bat algorithm has converged very quickly and gives the accurate result for prediction of best feature. There are multiple reasons for the success of bat-based algorithms. By analyzing the main element features and upgrading equations, we can summarize the next three key factors features: rate of recurrence tuning, bat algorithm uses echolocation and rate of recurrence tuning to resolve problems. Though echolocation is not used to imitate the real function the truth is immediately frequency variations are utilized. This capability provides some functionality like the key feature found in particle swarm search engine optimization. Therefore, bat algorithm has got the features of other swarmintelligence-based algorithms.

Auto zooming: In this bat algorithm has a definite benefit over other metaheuristic algorithms. That is, it has a capacity for automatically zooming into and where promising alternatives have been found. This zooming is associated with the automatic change from explorative techniques to local rigorous exploitation. As a total result, bat algorithm has an instant convergence rate, at least at first stages of the iterations, weighed against other algorithms.

Parameter control: Many metaheuristic algorithms used to set guidelines by using some, pre-tuned algorithm-dependent variables. On the other hand, bat algorithm uses parameter control, which may differ the ideals of variables ( $A$ and $r$ ) as the iterations continue and this provides ways to automatically change from exploration to exploitation when the perfect solution is nearing. In addition, initial theoretical evaluation recommended that this algorithm has assured global convergence properties under the right condition, and it can solve large-scale problems effectively also. The bat algorithm steps are given below.

1. Determine the fitness function in order to create bat inhabitants randomly.

2. Define pulse rate occurrence, declare the factors and parameters value for the pulse, loudness, and frequency as $(r, A, f)$.

3. Perform iteration: using while loop where $\left(t<T_{\max }\right)$

4. New solution is made by frequency modification, and

5. upgrading velocities and locations alternatives [Eq.(5) and Eq.(6) are used]

6. if $\left(\right.$ rand $\left.>\underline{r}_{i}\right)$

a. Decide on a solution one of the better solutions

b. Generate a local area solution throughout the best solution.

7. end if 
8. Use the bat flying randomly so that new solution can be generated.

9. if (rand $<A_{i}$ and $f\left(x_{i}\right)<f(x)$ )

a. It increases the value for $r_{i}$ (pulse rate) and reduces the loudness $\left(A_{i}\right)$, a new solution is found for pulse rate and loudness.

10. End if

11. Get ranking the bats and discover the new current best solution.

12. End of the loop and the result is displayed.

\section{EFFICIENT FUZZY KOHONEN CLUSTERING NETWORK (EFKCN)}

The efficient Fuzzy Kohonen Clustering Network (EFKCN) clustering is closely related to the Fuzzy C-Means (FCM) algorithms. The integration of FCM and Kohonen Clustering Network $(\mathrm{KCN})$ is one way to address several problems of $\mathrm{KCN}$. Here combine the ideas of fuzzy membership values for learning rates, and with the help of this learning, rate updates the cluster centre. This cluster centre is taken as the weight in the $\mathrm{KCN}$ algorithm. The essential ideas of FCM and Kohonen neural network combined with a fresh category of algorithms called Fuzzy Kohonen Clustering Network. Kohonen Clustering Network (KCN) clustering is meticulously related to the Fuzzy cMeans (FCM) algorithms Since Fuzzy c-Means algorithms are optimization procedure because the target function is approximately minimized. The integration of FCM and KCN is one way to handle several problems of $\mathrm{KCN}$. They incorporate the ideas of fuzzy membership values for learning rates, the parallelism of Fuzzy c-Means, and update guidelines of $\mathrm{KCN}$. FKCN is the self-organizing algorithm, because the size of the up to date area is automatically fine-tuned during learning, and FKN usually terminates, so of reduced purpose usually function of FCM. Fuzzy Kohonen clustering network systems (FKCN) are popular for clustering evaluation (unsupervised learning and selforganizing). This classification of FKCN algorithm is a couple of iterative techniques of strategies that undergo some major problems, for example, its contingency rate is not too fast for a sizable amount of datasets. To conquer these defects, a competent Efficient Fuzzy Kohonen Network (EFKN) algorithm is suggested in this paper, which can significantly decrease the computation time necessary to partition a dataset into desired clusters. By presenting the threshold ideals and fuzzy convergence providers in the network learning technique to change the training rates dynamically, the network convergence rate is greatly increased and improved upon and the problem rates of dataset cluster are significantly reduced. Experimental results show the new algorithm is normally $3 x$ faster than the initial FKCN algorithm. We also illustrate that the grade of the increased EFKCN is preferable to the initial FKCN algorithm. The algorithm of EFKCN is summarized as follows:

- Take the sample spaces as $X=\left(x_{1}, x_{2}, \ldots, x_{n}\right)$ and the threshold error $\varepsilon>0$ some small positive constant.

- The weight vector $V v_{0}=v_{10}, v_{20}, \ldots, v_{c 0}$, selects $m_{0}>1$ and iteration limit $\left(t_{\max }\right)$ are initialized.

For $t=1,2 \ldots, t_{\max }$ is taken to find the learning rates as shown. a. Find all learning rates:

$$
m_{t}=m_{0}-\mathrm{t}^{*} \Delta m, \Delta m=1 / \mathrm{t}_{\max }
$$

where, $m_{t}$ is the fuzzy membership for the $t$ iteration and $\Delta m$ is the fuzzy membership differences for each iteration for each member, update its membership function:

$$
u_{i k, t}=\left(\sum\left(\frac{\left\|X_{k}-V_{i, t-1}\right\|}{\left\|X_{k}-V_{j, t-1}\right\|}\right)^{\frac{2}{m-1}}\right)^{-1}
$$

where, $u_{i k, t}$ is the membership function of the $k^{\text {th }}$ data of $i^{\text {th }}$ cluster for each $t$ iteration; $X_{k}$ is the $k^{\text {th }}$ data; $V_{i, t-1}$ is the $i^{\text {th }}$ cluster center for $t-1$ iteration; $V_{j, t-1}$ is the $j^{\text {th }}$ cluster center for $t-1$ iteration and $m$ is weighting exponent on each fuzzy membership.

Compute learning rate

$$
\alpha_{t x, \tau}= \begin{cases}\left(u_{i k, t}\right)^{m_{u}} & u_{i k, t}>t_{d} \\ \left(u_{i k, t}\right)^{m_{t}} & t_{d} \leq u_{i k, t} \leq t_{u} \\ \left(u_{i k, t}\right)^{m_{d}} & u_{i k, t}<t_{d}\end{cases}
$$

where, $t_{u}$ and $t_{d}$ are threshold value, $t_{u}$ is upper cut set $(0.5,1), t_{d}$ is lower cut set $(0,0.5), m_{u}$ and $m_{d}$ are fuzzy convergence operators, $m_{u}(0,1)$ and $m_{d}>m_{t}$

b. Update all weight vectors:

$$
v_{i, t}=v_{i, t-1}+\frac{\sum_{k=1}^{n} \alpha_{i k, t}\left(x_{k}-v_{i, t-1}\right)}{\sum_{j=1}^{n} \alpha_{i j, t}}
$$

where, $v_{i}$ is $i^{\text {th }}$ cluster center and $v_{i, t}$, is $i^{\text {th }}$ cluster center for $t$ iteration

Compute the function

$$
E_{t}=\left\|V t-V_{t-1}\right\|
$$

If $t E_{t}<\epsilon$ stop, else $t=t+1$ goto update membership function. From this clustering process, the number of clusters results is given as input to SADM-ELM classifier. In this classifier the input neurons are obtained from this clustering result.

\section{CLASSIFICATION}

Classification plays a major role in diagnoses of the human heart disease. An accurate ECG signal classification is important task to identify the condition of the patient as low, medium or in high risk. In this work, 5 classes are taken to predict the disease of the patient. The classes taken are NSR, PVC, AF, VF, and BII. The extracted feature set is presented to the classifier to obtain the accurate result. Based on the classification the result shows the accuracy, specificity, sensitivity, F-measure, execution time is low when compared with the previous work and presented in the preceding section

\subsection{SADE-ELM CLASSIFIER}

Many machine learning algorithms are available for the classification of the diseases. In this paper, the popular machine learning algorithm, ELM is taken for the classification which combines the evolutionary algorithm uses the differential 
evolution in ELM to find the optimal solution. Basically, ELM is based on empirical risk minimization theory and it is fast in learning rates.

Even though, it provides better performances, robustness and generalization ability it lacks in over-fitting problem. To overcome this problem in the proposed work the ELM algorithm is combined with Evolutionary algorithm and named as Self Adaptive Differential Evolutionary SADE ELM to predict the classification result. The learning algorithm ELM, randomly generate neurons with the weight $\mathrm{w}$, bias $\mathrm{b}$ between input layer and hidden layer. It finds the neuron activation in the hidden layer and the output matrix $\mathrm{H}$ is calculated, with this it calculates the output weight.

The ELM produces the insight weights and concealed biases arbitrarily which will be the basis of determining the result weights, arbitrarily and might not exactly reach the optimal solution for classification. The proposed work is enhanced by integrating the ELM with as Self Adaptive Differential Evolutionary to optimize the input weights and hidden biases can obtain better generalization performance than ELM algorithm. This work proposes an SADE-ELM predicated on EFKCN clustering, which doesn't need to determine the amount of concealed nodes physically and arbitrarily.

The proposed algorithm automatically determines the amount of hidden nodes for different data sets. The suggested algorithm decides the amount of concealed nodes for different data models automatically. Every individual in the first generation made randomly which comprises the input weights and hidden biases: Secondly, the related matching result weights matrix for every single individual is determined and computed. The task of the SADE-ELM algorithm procedure is listed below.

\subsection{PROCEDURE}

1. Training set data is given as input to assess.

2. The parameter value $N P$ (size of the population is given).

3. It yields the classification result as output.

4. Generate a random initial population.

5. Measure the fitness value for each and every specific set with training collection data.

6. Use While-do loop until the condition is achieved randomly.

7. By using for loop (for $i=1$ to $N P$ do) create mutation vector $v_{i}$, by mutation procedure.

8. Create crossover vector $u_{i}$, by mutation process.

9. Find the superior individuals by selection procedure.

10. End of for loop;

11. End of while loop;

12. Measure the optimized model by evaluation set using ELM procedure.

13. Training set of heart rate data is given as $N=$ kernel function $f(x)$, and hidden neuron.

14. Select suitable activation function and number of hidden neurons.

15. Assign arbitrary input weight and bias.
16. Calculate the output matrix at the hidden layer and predicts the output weight.

\section{RESULT AND DISCUSSION}

The MIT-BIH arrhythmia database is employed as the data source in this study the database contains 48 recordings. Each has the length of time of thirty minutes and includes two leads; the altered limb lead II and one of the leads V1, V2, V4 or V5. The sampling rate occurrence is $360 \mathrm{~Hz}$, this information is band-pass filtered at $0.1-100 \mathrm{~Hz}$ and the quality is 200 examples per $\mathrm{mV}$. Twenty-three of the recordings are designed to serve as the sample of regular medical recordings and 25 recordings sophisticated ventricular, junction, and supraventricular arrhythmias.

We can discover a couple of over 109,000 labelled ventricular beats from 15 different heartbeat types. There is a huge difference in the number of examples in each heart beat type. The most significant class is Normal beat with about 75,000 examples and the tiniest class is Supraventricular premature beat (SP) with just two examples. The data repository is indexed both in timing information and classification. For additional information about MITBIH Arrhythmia database see [20], a total was utilized by us of 12 records from the database. We extracted a complete of 30,873 beats; 22,476 normal beats, 5,394 irregular PVC arrhythmia beats, and 3,003 other arrhythmic beats. The database was utilized by us as index files from the database to locate beats in ECG signals.

The following values are used to find the Accuracy, specificity, Sensitivity, F-measure, execution time and mean error rate $T N, T P, F N, F P$. TP - True Positive, $T N$ - True Negative, FP - False Positive, $F N$ - False Negative. The purpose of identifying the result of $T N, T P, F N$ and $F P$ is

- TP: sick individuals properly known as sick

- FP: healthy individuals incorrectly known as sick

- TP: healthy individuals properly known as healthy

- FN: sick individuals incorrectly known as healthy

Including the suggested work classifies with these five classes such as normal sinus rhythm (NSR), premature ventricular contraction (PVC), Atrial Fibrillation (AF), Ventricular Fibrillation (VF) and $2^{\circ}$ block (BII). If for example a portion of HRV with the VF arrhythmia is categorized as the VF, then it is stated that the portion is labelled TP. Alternatively, in case a nonVF segment is classified as non-VF, then it is stated that the segment is classified TN. Any non-VF segment which is classified a VF segment in error will create an FP. while any VF segment which is classified a non-VF segment in error will create an FN result. While any VF section which is categorized a nonVF section in error will create an FN effect. For the analysis of proposed classifier, a complete of 1317 segments, that are obtained from the MIT-BIH arrhythmia database, were used and it contains 835 NSR segments, 57 PVC segments, $322 \mathrm{AF}$ segments, $78 \mathrm{VF}$ segments and $25 \mathrm{BII}$ segments. The execution experimented on a number of datasets and results offered in Table.1 represent the performances. The Accuracy, specificity, Sensitivity, F-measure, execution time and mean error rate is shown in Fig.3 to Fig.8. 


\subsection{ACCURACY COMPARISON}

Accuracy is thought as

$$
\text { Accuracy }=\frac{T N+T P}{T P+T N+F N+F P}
$$

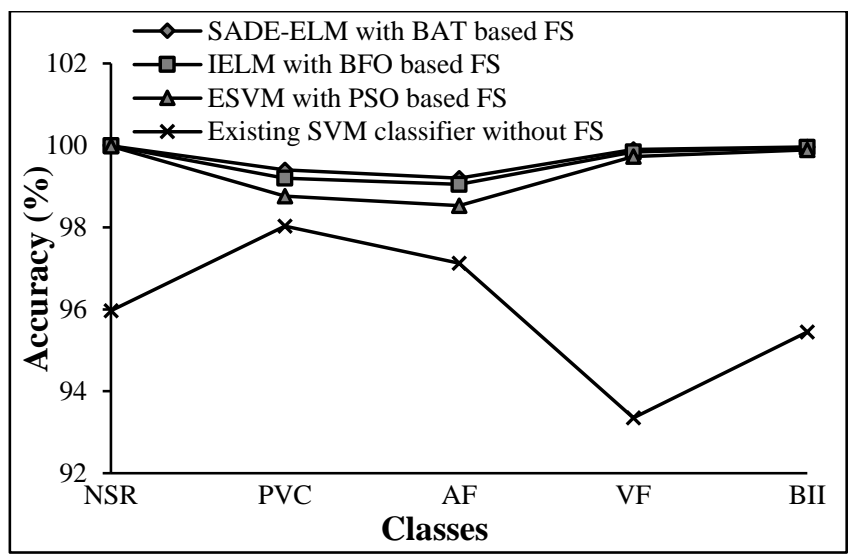

Fig.3. Accuracy Comparison

\subsection{SENSITIVITY COMPARISON}

Sensitivity identifies the test's capability to properly discover or find patients who do have the problem. Within the exemplary case of a medical test used to recognize an illness, the level of sensitivity of the test is the percentage of patients who test positive for the condition. Mathematically, this is expressed as:

$$
\text { Sensitivity }=\frac{\text { No. of TP }}{\text { No. of } T P+\text { No. of } F N}
$$

where, number of true positive/total number of sick patient, gives the probability of a positive test results that the patient has the disease.

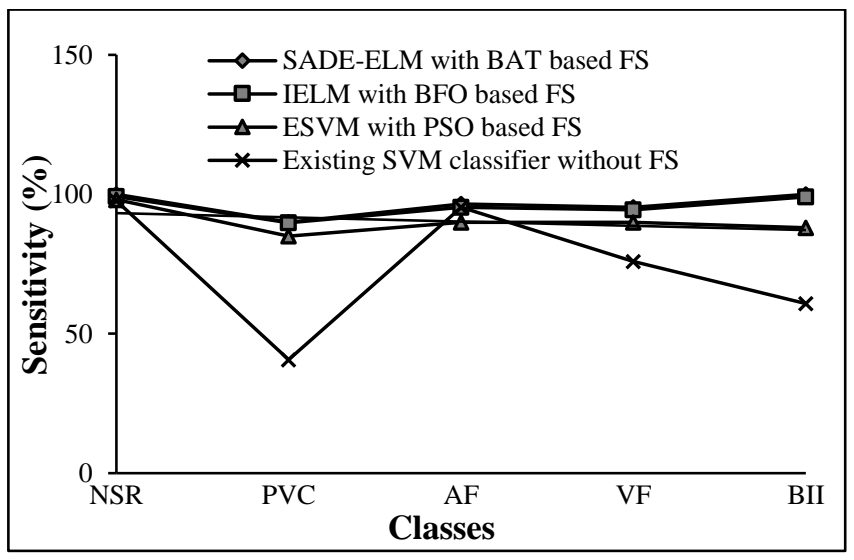

Fig.4. Sensitivity Comparison

\subsection{SPECIFICITY COMPARISON}

Specificity pertains to the test's potential to appropriately identify patients without a condition. Consider the exemplary case of a medical test for diagnosing an illness. Specificity of the patient test is the percentage of healthy patients does not known to have the condition and test results appears negative. This can be written mathematically as:

$$
\text { Specificity }=\frac{\text { No. of } T N}{\text { No. of } T N+\text { No. of FP }}
$$

This gives the number of TN/total number of well individual in the populations where the probability of the test results that the patient is well.

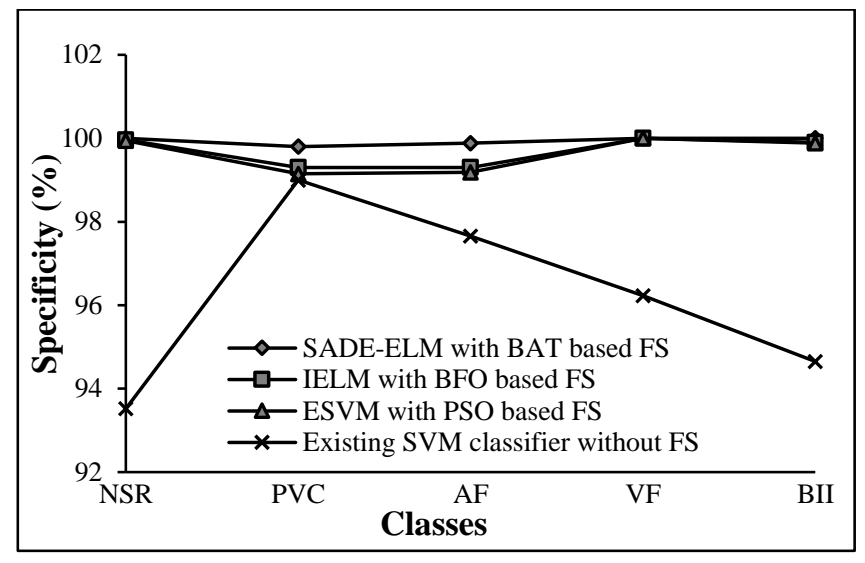

Fig.5. Specificity Comparison

\subsection{F-MEASURE COMPARISON}

In classification the F-measure is accuracy test, which has both $P$ and $R . P$ is precision, the number of correct positive result divided by the number of all positive result. $R$ is the recall, the number of correct positive result divided by the number of all positive result. Hence this $F$-measure calculates the average value of $P R$, and it predicts the best value if it is 1 and 0 if it is worst value. $F 1$ strategy is thought as the harmonic mean of accuracy and recall. An excellent classifier is assumed to truly have a high $F 1$ measure, which indicates that the classifier performs well regarding both precision $(P)$ and recall $(R)$.

$$
F 1 \text { strategy }=(2 P R) /(P+R)
$$

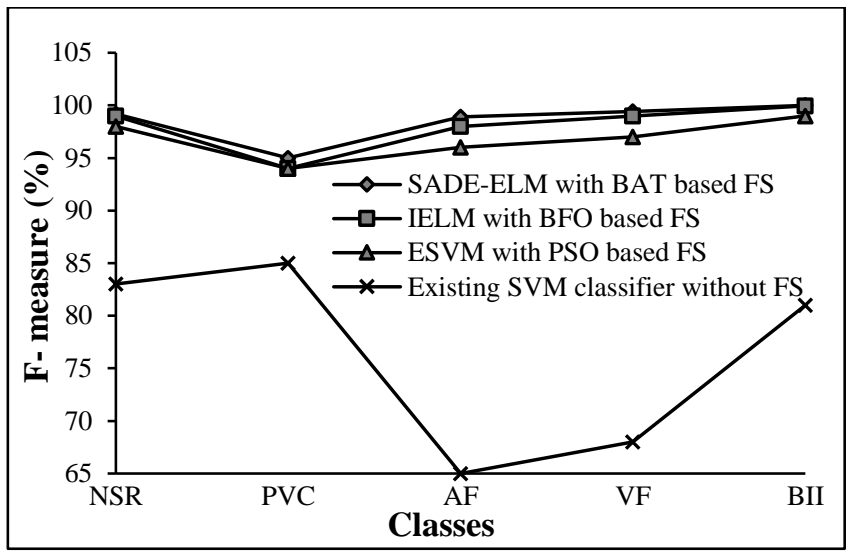

Fig.6. F-Measure Comparison

\subsection{EXECUTION TIME COMPARISON}

The execution time shows that the proposed algorithm gives the result at the earliest compared with the previous work.

The mean absolute error MAE is the amount used to assess and evaluate how the value is predicted and reveals the final result. It is given by Eq.(20) 


$$
M A E=\frac{1}{n} \sum_{i=1}^{n}\left|f_{i}-y_{i}\right|=\frac{1}{n} \sum_{i=1}^{n}\left|e_{i}\right|
$$

As the name suggests, the mean absolute error is an average of the absolute errors, where are the prediction and the true value

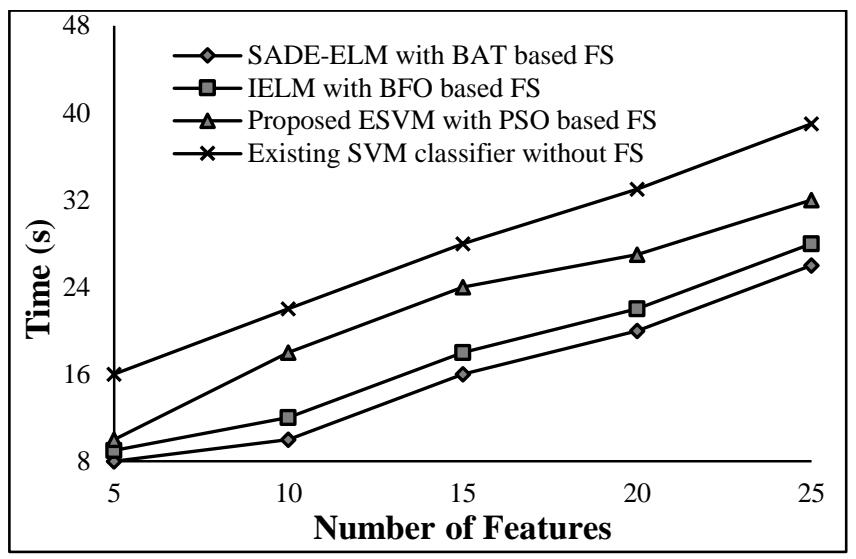

Fig.7. Execution Time Comparison

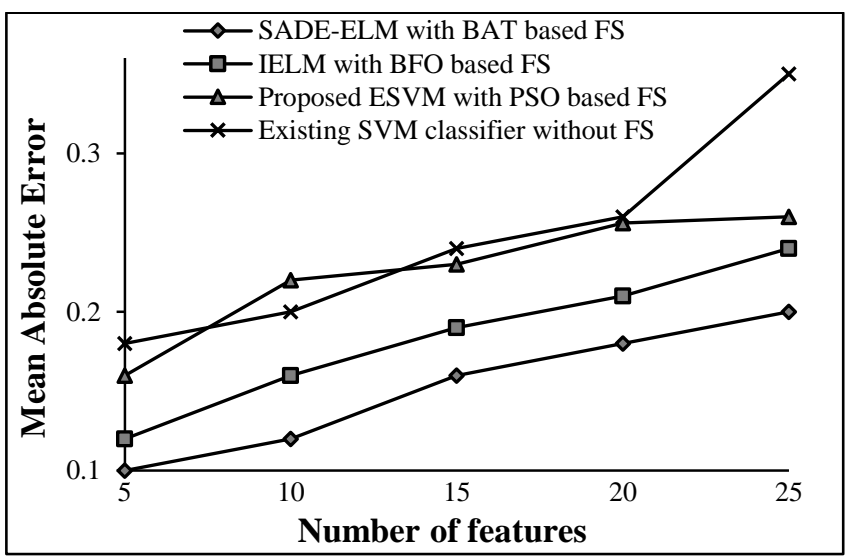

Fig.8. Mean Absolute error

Table.1. Accuracy for each class (\%) for SADE-ELM method compared with previous methods.

\begin{tabular}{|l|c|c|c|c|c|}
\hline \multicolumn{1}{c|}{$\begin{array}{c}\text { Classes/ } \\
\text { Algorithm }\end{array}$} & NSR & PVC & AF & VF & BII \\
\hline SVM classifier without FS & 95.97 & 98.03 & 97.13 & 93.35 & 95.45 \\
\hline ESVM with PSO based FS & 99.98 & 98.76 & 98.53 & 99.73 & 99.89 \\
\hline IELM with BFO based FS & 99.09 & 99.2 & 99.05 & 99.84 & 99.95 \\
\hline $\begin{array}{l}\text { SADE-ELM with BAT } \\
\text { based FS }\end{array}$ & 99.99 & 99.4 & 99.21 & 99.9 & 99.96 \\
\hline
\end{tabular}

\section{CONCLUSION}

The very best accuracy rate is achieved using the linear and non-linear feature vector and the Bat based feature selection algorithm only using less quantity of selected features and the classification is performed using SADE-ELM based EFKCN is significantly reduced the computational complexity. With the purpose of improving the classification precision in HRV transmission, in this paper, we propose a fresh classifier method included the self-adaptive differential evolution extreme learning machine specifically SADE-ELM algorithm. The SADE-ELM classifier is offered as the classifier to classify the heart rate data. Furthermore, SADE- ELM algorithm can solve the complicated non-linear relationship between influencing signals and the standard of HRV transmission, and it can get higher prediction precision in the analysis. To evaluate the performance of this suggested method, it can be employed to equate to IELM-BFO, SVM, and ESVM-PSO algorithms. The experimental results illustrate the suggested method SADE-ELM algorithm would work and effective for classifying the heart rate data and increase the precision of HRV transmission. The suggested SADE-ELM classifier demonstrated and shows in discriminating five types of arrhythmia with BAT feature selection algorithm with EFKCN clustering. The precision, accuracy, and reliability of discrimination of NSR, PVC, AF, BII and VF were $99.99 \%$, $99.4 \%, 99.2 \%, 99.9 \%$, and $99.96 \%$, respectively. Future research will be aimed towards generalized discriminates methods which are being used to equate to the strategy of SADE-ELM and concentrate in other conditions such as classification velocity and computational cost.

\section{REFERENCES}

[1] R. Chary et.al., "Classification of Cardiac Abnormalities using Heart Rate Signals", Medical and Biological Engineering and Computing, Vol. 42, No. 3, pp. 288-293, 2005.

[2] M.H. Song et.al., "Support Vector Machine Based Arrhythmia Classification using Reduced Features", International Journal of Control, Automation and Systems, Vol. 3, No. 4, pp. 571-579, 2005.

[3] M.G. Tsipouras, Y. Goletsis and D.I. Fotiadis, "A Method for Arrhythmic Episode Classification in ECGs using Fuzzy Logic and Markov Models", Computers in Cardiology, pp. 361-364, 2004.

[4] M.G. Tsipouras, D.I. Fotiadis and D. Sideris, "An Arrhythmia Classification System based on the RR-Interval Signal", Artificial Intelligence In Medicine, Vol. 33, No. 3, pp. 237-250,2005.

[5] Carlos W.D. de Almeida, Renata M.C.R. Souza and Ana Lucia B. Candeias, "IFKCN: Applying Fuzzy Kohonen Clustering Network to Interval Data", Proceedings of International Joint Conference on Neural Networks, pp. 16, 2012.

[6] Jinhui Fan, Songmin Jia and Xiuzhi Li, "The Application of Fuzzy Kohonen Clustering Network for Intelligent Wheelchair Motion Control", Proceedings of IEEE International Conference on Robotics and Biomimetics, pp. 1-6, 2013.

[7] J Spilka, V Chudacek, J Kuzilek, L Lhotska and M Hanuliak, "Detection of Inferior Myocardial Infarction: A Comparison of Various Decision Systems and Learning Algorithms", Computing in Cardiology, pp. 273-276, 2010.

[8] H.K. Chatterjee, R. Gupta and M. Mitra, "A Statistical Approach for Determination of Time Plane Features from Digitized ECG", Computers in Biology and Medicine, Vol. 41, No. 5, pp. 278-284, 2011.

[9] Iztok Fister, Xin-She Yang, Simon Fong and Yan Zhuang, "Bat algorithm: Recent advances", Proceedings of IEEE $15^{\text {th }}$ International Symposium on Computational Intelligence and Informatics, pp. 163-167, 2014. 
[10] Swati Banerjee and Madhuchhanda Mitra, "Classification of ST and Q Type MI Variant using Thresholding and Neighbourhood Estimation Method after Cross Wavelet based Analysis", Cornell University Library, pp. 1-14, 2013.

[11] Li Sun, Yanping Lu, Kaitao Yang and Shaozi Li, "ECG Analysis using Multiple Instance Learning for Myocardial Infarction Detection", IEEE Transactions on Biomedical Engineering, Vol. 59, No. 12, pp. 3348-3356, 2012.

[12] Marco Dorigo, Mauro Birattari and Thomas Stutzle, "Ant Colony Optimization”, IEEE Computational Intelligence Magazine, Vol. 1, No. 4, pp. 28-39, 2006.

[13] James Kennedy, "Particle Swarm Optimization", Springer, 2010.

[14] Padmavathi Kora and Sri Ramakrishna Kalva, "Hybrid Bacterial Foraging and Particle Swarm Optimization for
Detecting Bundle Branch Block", SpringerPlus, Vol. 4, pp 1-19, 2015.

[15] Xin-She Yang, "A New Metaheuristic Bat-Inspired Algorithm", Proceedings of Nature Inspired Cooperative Strategies for Optimization, Vol. 284, pp. 65-74, 2010.

[16] Xin-She Yang and Xingshi He, "Bat Algorithm: Literature Review and Applications", International Journal of BioInspired Computation, Vol. 5, No. 3, pp.141-149, 2013.

[17] Iztok Fister Jr, et al., "Particle Swarm Optimization for Automatic Creation of Complex Graphic Characters", Chaos, Solitons and Fractals, Vol. 73, pp. 29-35, 2015.

[18] Xin-She Yang, "Bat Algorithm for Multi-Objective Optimisation", International Journal of Bio-Inspired Computation, Vol. 3, No. 5, pp. 267-274, 2011. 